\title{
Biodegradable Blend Nanoparticles of Amphiphilic Diblock Copolymers Prepared by Nano-Precipitation Method
}

\author{
Wichuda Nanthakasri, Mangkorn Srisa-Ard, Yodthong Baimark* \\ Department of Chemistry and Center of Excellence for Innovation in Chemistry, Faculty of Science, Mahasarakham University, \\ Mahasarakham, Thailand. \\ E-mail: *yodthong.b@msu.ac.th, ²yodthongb@gmail.com
}

Received September $30^{\text {th }}$, 2011; revised November $15^{\text {th }}$, 2011; accepted November $26^{\text {th }}, 2011$.

\begin{abstract}
Nanoparticles of biodegradable methoxy poly(ethylene glycol)-b-polyester amphiphilic diblock copolymers have widely investigated for use as controlled release drug delivery carriers. In this work, blend nanoparticles of methoxy poly(ethylene glycol)-b-poly(D,L-lactide) (MPEG-b-PDLL) and methoxy poly(ethylene glycol)-b-poly(e-caprolactone) (MPEG$b-P C L)$ were prepared by nano-precipitation method without any surfactants. ${ }^{1} \mathrm{H}-N M R$ spectra showed significant difference in integral peak areas, suggesting the nanoparticles with different MPEG-b-PDLL/MPEG-b-PCL blend ratios can be prepared. Transmission electron microscope revealed the blend nanoparticles had nearly spherical in shape with smooth surface. Average size of the blend nanoparticles obtained from light-scattering analysis slightly decreased with increase in blend ratio of MPEG-b-PCL. The MPEG-b-PDLL and MPEG-b-PCL were amorphous and semi-crystalline, respectively. Thermal transition properties of the blend nanoparticles were studied with differential scanning calorimetry (DSC). The DSC results showed that glass transition temperatures of the blend nanoparticles decreased and heats of melting steadily increased, while the melting temperature did not change as the MPEG-b-PCL blend ratio increased. This indicates the miscibility of MPEG-b-PDLL and MPEG-b-PCL in the amorphous phase of the blend nanoparticles. Thermogravimetric analysis showed that the blend nanoparticles clearly exhibited two thermal decomposition steps due to MPEG-b-PDLL decomposition followed with MPEG-b-PCL. The blend nanoparticles had two temperatures of maximum decomposition rate $\left(T_{d, \max }\right)$ accorded to each blend component. The $T_{d, \max }$ of MPEG-b$P D L L$ phase significantly decreased, while $T_{d, \max }$ of MPEG-b-PCL phase did not change as the MPEG-b-PCL blend ratio increased. These results suggested that the desired thermal properties of blend nanoparticles can be tailored by varying the blend ratio.
\end{abstract}

Keywords: D,L-Lactide, e-Caprolactone, Diblock Copolymers, Blend Nanoparticles, Nano-Precipitation

\section{Introduction}

Amphiphilic diblock copolymer nanoparticles of methoxy poly(ethylene glycol)-b-poly(D,L-lactide) (MPEG- $b$ PDLL) and methoxy poly(ethylene glycol)-b-poly( $\varepsilon$ caprolactone) (MPEG-b-PCL) have attracted much attention in drug delivery applications in the human body due to their biocompatibility and biodegradability [1-4]. The hydrophilic MPEG layer coated on the nanoparticle surfaces can increase blood circulation time of these nanoparticles [5,6]. Their thermal, biodegradation and drug release properties can be controlled through several approaches including block length adjustment [7-10] and copolymerization [11]. The physical blending is an al- ternative method that has been widely used to adjust the properties of polymers such as crystallinity, mechanical properties and biodegradation properties [12-15]. These properties strongly affected the drug release behaviors.

In addition, the MPEG segments attached to polyester segments can improve miscibility between PDLL and PCL blends to reduce the phase separation [12]. Thus, unique properties of polymer blends quite different from their origin polymers were obtained. However, the preparation of blend nanoparticles of the amphiphilic diblock copolymers has been scarcely published.

The present work describes the preparation of blend nanoparticles of amorphous MPEG- $b$-PDLL and semi- 
crystalline MPEG-b-PCL. The blend nanoparticle were prepared by nano-precipitation method, which were then investigated by transmission electron microscopy (TEM) for particle morphology, ${ }^{1} \mathrm{H}-\mathrm{NMR}$ spectroscopy for chemical composition and light-scattering analysis for particle size and size distribution. Thermal properties of the blend nanoparticles were also determined by differential scanning calorimetry (DSC) and thermogravimetric analysis (TGA).

\section{Materials and Methods}

\subsection{Materials}

D,L-lactide (DLL) monomer was synthesized by thermal decomposition reaction of low molecular weight poly (D,L-lactic acid) (PDLLA) at $220^{\circ} \mathrm{C}$ under reduced pressure. The low molecular weight PDLLA was obtained from polycondensation of D,L-lactic acid solution $(90 \%$, Fluka) at $160^{\circ} \mathrm{C}$. Crude DLL was purified by repeated re-crystallization from ethyl acetate and dried in vacuo at $50^{\circ} \mathrm{C}$ for $48 \mathrm{~h}$ before use. $\varepsilon$-Caprolactone (CL) monomer (99\%, Acro, USA) was purified by drying with $\mathrm{CaH}_{2}$ followed by distillation under reduced pressure before storage over molecular sieves in a refrigerator. Methoxy polyethylene glycol (MPEG) with a molecular weight of $5000 \mathrm{~g} / \mathrm{mol}$ (Fluka) was used after it was dried in vacuo at $120^{\circ} \mathrm{C}$ for $4 \mathrm{~h}$. Stannous octoate (Sn(Oct) $)_{2}, 95 \%$ Sigma) was used without further purification. All solvents in analytical grade were used.

\subsection{Synthesis of Diblock Copolymers}

Both MPEG- $b$-PDLL and MPEG- $b$-PCL diblock copolymers were synthesized by ring-opening polymerization in bulk under nitrogen atmosphere at $130^{\circ} \mathrm{C}$ for $24 \mathrm{~h}$. Feed mole ratios of MPEG/DLL and MPEG/CL were $1 / 416$ and 1/526, respectively. MPEG and Sn(Oct) $)_{2}$ were used as the initiating system. $\mathrm{Sn}(\mathrm{Oct})_{2}$ concentration was kept constant at $0.02 \mathrm{~mol} \%$. The diblock copolymers were dissolved in chloroform before precipitating in cool $n$-hexane for purification. They were then dried to constant weight in vacuo at room temperature. According to this procedure, the purified diblock copolymers were obtained with more than $95 \%$ yields.

\subsection{Characterization of Diblock Copolymers}

\subsection{1. ${ }^{1} \mathrm{H}-\mathrm{NMR}$ Spectrometry}

Chemical compositions of the copolymers were measured by ${ }^{1} \mathrm{H}$-NMR spectrometry using a Bruker Advance DPX $300{ }^{1} \mathrm{H}$-NMR spectrometer at $25^{\circ} \mathrm{C} \mathrm{CDCl}_{3}$ was used as the solvent, and tetramethysilane was used as the internal standard.

\subsubsection{Gel Permeation Chromatography}

The number-average molecular weight $\left(M_{n}\right)$ and molecu- lar weight distribution $(M W D)$ of the copolymers were determined by gel permeation chromatography (GPC) using a Waters 717 plus Autosampler GPC equipped with an Ultrastyragel ${ }^{\circledR}$ column operating at $35^{\circ} \mathrm{C}$ and employing a refractive index detector. Tetrahydrofuran was used as the solvent at a flow rate of $1 \mathrm{~mL} / \mathrm{min}$.

\subsubsection{Differential Scanning Calorimetry}

Thermal transition properties of the copolymers were carried out by non-isothermal differential scanning calorimetry (DSC) using a Perkin-Elmer Pyris Diamond DSC The sample $(\sim 10 \mathrm{mg})$ was heated at the rate of $10^{\circ} \mathrm{C} / \mathrm{min}$ under helium flow.

\subsection{Preparation of Blend Nanoparticles}

Blend nanoparticles of diblock copolymers were prepared by the nano-precipitation method. Briefly, $60 \mathrm{mg}$ mixed copolymer was dissolved in $6 \mathrm{~mL}$ acetone. The blend solution was added drop-wise into $60 \mathrm{~mL}$ distilled water in a $100 \mathrm{~mL}$ beaker with magnetic stirring at speed of $800 \mathrm{rpm}$. The nanoparticle colloid was obtained after evaporating acetone at room temperature for $6 \mathrm{~h}$ in a fume hood. The nanoparticle aggregates were removed by centrifugation at $5000 \mathrm{rpm}$ at $4^{\circ} \mathrm{C}$ for $30 \mathrm{~min}$. The blend nanoparticles with MPEG- $b$-PDLL/MPEG- $b$-PCL blend ratios of 4/0,3/1, 2/2, 1/3 and 0/4 (w/w) were prepared. Dried nanoparticle sample was collected by centrifugation at 15,000 at $4^{\circ} \mathrm{C}$ for $2 \mathrm{~h}$ before freeze-drying for overnight.

\subsection{Characterization of Blend Nanoparticles}

\subsubsection{Transmission Electron Microscopy}

Morphology of the blend nanoparticles was determined by transmission electron microscopy (TEM) using a JEOL JEM 1230 TEM. For TEM analysis, a drop of nanoparticle suspension was placed on a formvar film coated on the copper grid. The specimen on the copper grid was not stained.

\subsubsection{Light-Scattering Particle Size Analysis}

Particle size and size distribution of the blend nanoparticles were measured from the nanoparticle colloids by light-scattering analysis using a Coulter LS230 particle size analyzer at $25^{\circ} \mathrm{C}$.

\subsection{3. ${ }^{1} \mathrm{H}$-NMR Spectrometry}

Chemical functional groups of the blend nanoparticles were studied by ${ }^{1} \mathrm{H}$-NMR spectrometry using a Bruker Advance DPX $300{ }^{1} \mathrm{H}$-NMR spectrometer at $25^{\circ} \mathrm{C}$. $\mathrm{CDCl}_{3}$ was used as the solvent, and tetramethysilane was used as the internal standard.

\subsubsection{Differential Scanning Calorimetry}

Thermal transition properties of the blend nanoparticles were determined by non-isothermal differential scanning 
calorimetry (DSC) as described above.

\subsubsection{Thermogravimetric Analysis}

The thermal decomposition behaviours of the blend nanoparticles were characterized by non-isothermal thermogravimetric (TG) analysis using a TA-Instrument SDT Q600 TG analyzer. For TG analysis, sample ( $\sim 5 \mathrm{mg})$ was heated from $50^{\circ} \mathrm{C}$ to $800^{\circ} \mathrm{C}$ at the rate of $20^{\circ} \mathrm{C} / \mathrm{min}$ under nitrogen atmosphere.

\section{Results and Discussion}

\subsection{Characterization of Diblock Copolymers}

Chemical compositions of diblock copolymers were determined from ${ }^{1} \mathrm{H}$-NMR spectra. For this purpose, the ratio of integral peak areas was calculated corresponding to the ethylene oxide (EO, repeating units of MPEG) methylene protons at $\delta=3.6-3.7 \mathrm{ppm}$, the DLL methine protons at $\delta=5.0-5.3 \mathrm{ppm}$ and the CL $\varepsilon$-methylene protons at $\delta=4.0-4.2 \mathrm{ppm}$. The chemical compositions of MPEG- $b$-PDLL and MPEG- $b$-PCL were measured as $\mathrm{EO} / \mathrm{DLL}=20 / 80$ and $\mathrm{EO} / \mathrm{CL}=18 / 82(\mathrm{~mol} \%)$ corresponding to the MPEG/DLL and the MPEG/CL mole ratios of $1 / 452$ and $1 / 515$, respectively. These calculated copolymer compositions are similar to MPEG/monomer (DLL or CL) feed mole ratios (1/416 and $1 / 526$ for MPEG- $b$-PDLL and MPEG- $b$-PCL, respectively). This suggests that the polymerization reactions were taken to near-quantitative conversion.

The $M_{n}$ s of MPEG- $b$-PDLL and MPEG- $b$-PCL obtained from GPC curves were 49,000 and 47,000 g/mol, respectively. The $M W D$ values were 1.8 and 1.6 for the MPEG- $b$-PDLL and the MPEG- $b$-PCL, respectively. The $M_{n}$ s obtained from GPC results were less than to that calculated from the feed ratios $(65,000 \mathrm{~g} / \mathrm{mol})$. The transesterification degradation side-reactions may occur during polymerization.

\subsection{Characterization of Blend Nanoparticles}

Figure 1 shows the TEM micrographs of various blend nanoparticles at different MPEG- $b$-PDLL/MPEG- $b$-PCL blend ratios. It can be clearly seen that the blend nanoparticles had nearly spherical in shape with smooth surface. They had nanometer in size range. The blend ratio did not affect on the morphology of nanoparticles. Average particle sizes of the blend nanoparticles measured from light-scattering analysis are summarized in Table 1. The size of MPEG- $b$-PCL nanoparticles was the smallest. The average sizes of blend nanoparticles increased significantly as the MPEG- $b$-PDLL blend ratio increased. This may be due to the crystallisable PCL block enhanced more compact nanoparticles.

The chemical compositions of blend components in the nanoparticles can be investigated from the ${ }^{1} \mathrm{H}-\mathrm{NMR}$

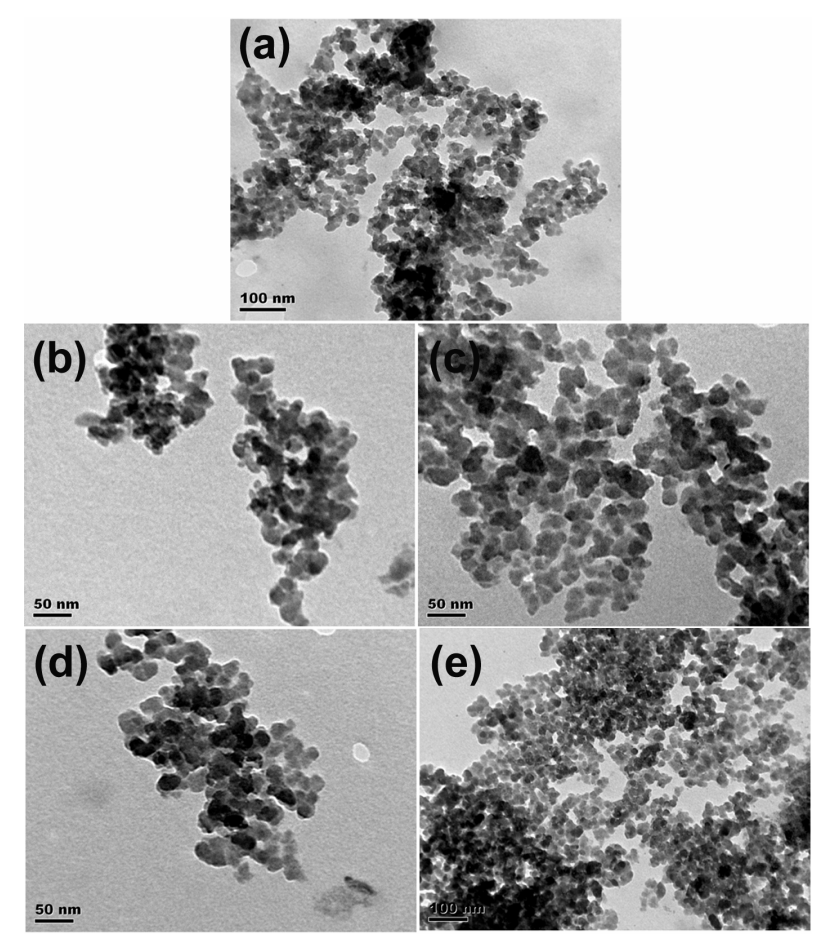

Figure 1. TEM micrographs of blend nanoparticles with MPEG- $b$-PDLL/MPEG-b-PCL blend ratios of (a) 4/0, (b) $3 / 1$, (c) $2 / 2$, (d) $1 / 3$ and (e) $0 / 4 \mathrm{w} / \mathrm{w}$. Bars $=100 \mathrm{~nm}$ for (a) and (e), and bars $=50 \mathrm{~nm}$ for (b)-(d).

Table 1. Average particle sizes and thermal properties of blend nanoparticles.

\begin{tabular}{cccccc}
\hline MPEG-b-PDLL/MPEG- $b$-PCL blend ratio $(\mathrm{w} / \mathrm{w})$ & Size $(\mathrm{nm})^{\mathrm{a}}$ & $T_{g}\left({ }^{\circ} \mathrm{C}\right)^{\mathrm{b}}$ & $T_{m}\left({ }^{\circ} \mathrm{C}\right)^{\mathrm{c}}$ & $\Delta H_{m}\left({ }^{\circ} \mathrm{C}\right)^{\mathrm{d}}$ & $T_{d, \max }\left({ }^{\circ} \mathrm{C}\right)^{\mathrm{e}}$ \\
\hline $4 / 0$ & $348 \pm 16$ & 36 & - & - & 367 \\
$3 / 1$ & $176 \pm 16$ & 34 & 48,55 & $6.9,2.9$ & 334,420 \\
$2 / 2$ & $162 \pm 16$ & 29 & 54 & 23.4 & 329,420 \\
$1 / 3$ & $98 \pm 15$ & 25 & 55 & 56.9 & 319,420 \\
$0 / 4$ & $98 \pm 18$ & - & 55 & 420 \\
\hline
\end{tabular}

${ }^{\mathrm{a}}$ Average particle size measured by light-scattering analysis; ${ }^{\mathrm{b}}$ Glass transition temperature obtained from DSC thermograms; ${ }^{\mathrm{c}}$ Melting temperature obtained from DSC thermograms; ${ }^{\mathrm{d}}$ Heat of melting obtained from DSC thermograms; ${ }^{\mathrm{e}}$ Temperature of maximum decomposition rate measured from DTG thermograms. 
spectra, as illustrated in Figure 2. It was found that the integral peak areas of DLL and CL units directly related to their blend ratios. The integral area of peak b (methine protons of DLL units) steadily decreased with decrease in the MPEG- $b$-PDLL blend ratio. Meanwhile the integral areas of peaks $\mathrm{d}$ and $\mathrm{h}$ (methylene protons of $\mathrm{CL}$ units) significantly increased as the MPEG- $b$-PCL blend ratio increased. This supported that the blend nanoparticles with different blend ratios can be prepared by nano-precipitation method of the blend solution.

The DSC thermograms of the MPEG- $b$-PDLL and MPEG- $b$-PCL showed that they were amorphous and semi-crystalline copolymers, respectively (DSC curves did not show). The MPEG- $b$-PDLL exhibited a single glass transition temperature $\left(T_{g}\right)$ at $36^{\circ} \mathrm{C}$. The melting temperature $\left(T_{m}\right)$ and heat of melting $\left(\Delta H_{m}\right)$ of MPEG$b$-PCL were $61^{\circ} \mathrm{C}$ and $86.0 \mathrm{~J} / \mathrm{g}$, respectively. The DSC thermograms of the blend nanoparticles are presented in Figure 3 and the DSC results are also summarized in Table 1. The DSC thermograms of blend nanoparticles exhibited both $T_{g}$ of MPEG- $b$-PDLL and $T_{m}$ of MPEG$b$-PCL. The $T_{g}$ of MPEG- $b$-PDLL in blend nanoparticles significantly decreased as the MPEG- $b$-PCL blend ratio

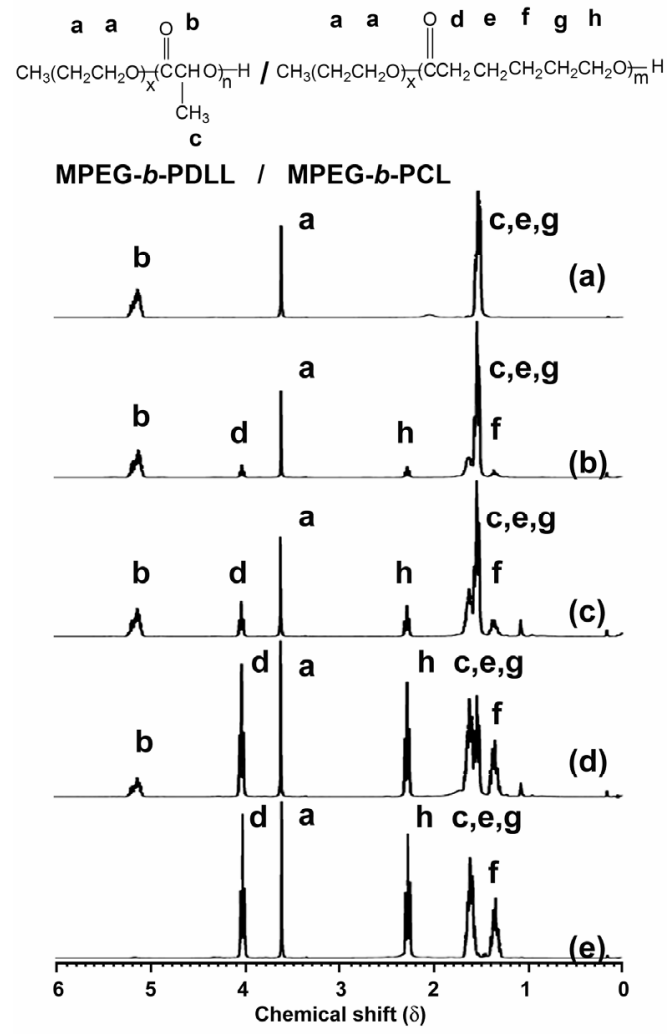

Figure 2. ${ }^{1} \mathrm{H}$-NMR spectra of blend nanoparticles with MPEG- $b$-PDLL/MPEG- $b$-PCL blend ratios of (a) 4/0, (b) $3 / 1$, (c) $2 / 2$, (d) $1 / 3$ and (e) $0 / 4 \mathrm{w} / \mathrm{w}$ (Peak-proton assignments as shown).

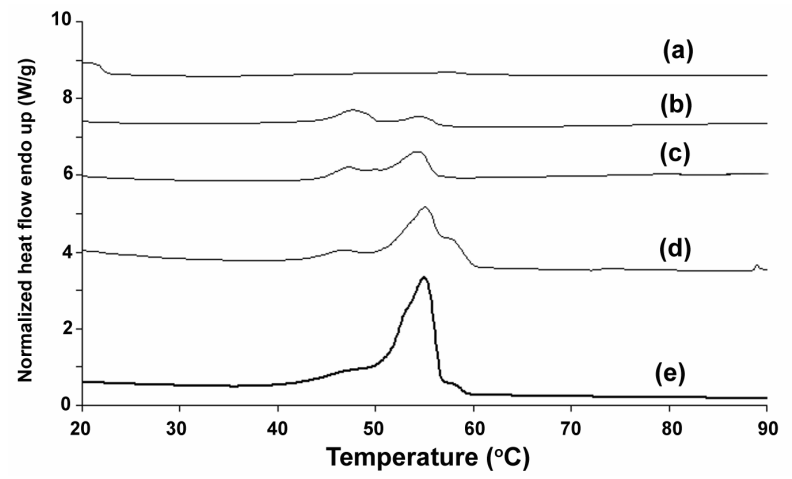

Figure 3. DSC thermograms of blend nanoparticles with MPEG- $b$-PDLL/MPEG- $b$-PCL blend ratios of (a) 4/0, (b) $3 / 1$, (c) $2 / 2$, (d) $1 / 3$ and (e) $0 / 4 \mathrm{w} / \mathrm{w}$ (heating rate $=10^{\circ} \mathrm{C}$ under helium flow).

increased, suggesting that the MPEG- $b$-PDLL and MPEG- $b$-PCL exhibited miscible blended in the amorphous phase to reduce the $T_{g}$ of MPEG- $b$-PDLL. The $T_{m}$ of MPEG- $b$-PCL in blend nanoparticles did not change. It should be noted that the $\Delta H_{m}$ values of the blend nanoparticles decreased steadily as increasing the MPEG$b$-PDLL blend ratio. This may be explained that the MPEG- $b$-PDLL interrupted crystallisability of MPEG- $b$ PCL in the blend nanoparticles.

The blend nanoparticles with controllable thermal transition properties $\left(T_{g}\right.$ and $\left.\Delta H_{m}\right)$ might be of interest for controlled release drug delivery applications. Two mains mechanisms of drug releasing from polymer matrix consisted of drug diffusion due to matrix swelling and drug release due to erosion of polymer matrix. The swelling and erosion rates of polymeric matrix strongly depended upon the $T_{g}$ and $\Delta H_{m}$, respectively. Thus drug release may be adjusted by varying the $T_{g}$ and $\Delta H_{m}$ of the polymeric matrix.

Thermal stability of the blend nanoparticles was determined from the TG thermograms, as shown in Figure 4. The weight losses of MPEG- $b$-PDLL and MPEG- $b$ PCL started at approximate $300^{\circ} \mathrm{C}$ and $350^{\circ} \mathrm{C}$, respectively. The MPEG- $b$-PCL exhibited higher thermal stability than that of MPEG- $b$-PDLL. The thermal decompositions completely finished at approximate $400^{\circ} \mathrm{C}$ and $450^{\circ} \mathrm{C}$ for the MPEG- $b$-PDLL and the MPEG- $b$-PCL, respectively. The blend nanoparticles showed two thermal decomposition stages with the first MPEG- $b$-PDLL decomposition following with the weight loss of MPEG$b$-PCL component. The weight loss behaviors of the blend nanoparticles directly related to the blend ratio. The weight remaining of the first decomposition step decreased steadily with the MPEG- $b$-PDLL blend ratio. The $2 / 2(w / w)$ MPEG- $b$-PDLL/MPEG- $b$-PCL blend nanoparticles showed remaining weight at approximately 


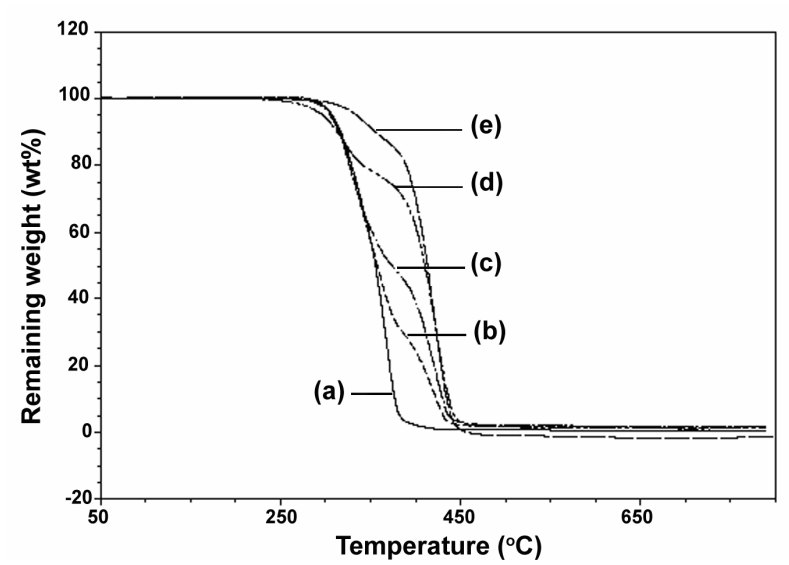

Figure 4. TG thermograms of blend nanoparticles with MPEG- $b$-PDLL/MPEG- $b$-PCL blend ratios of (a) $4 / 0$, (b) $3 / 1$, (c) $2 / 2$, (d) $1 / 3$ and (e) $0 / 4 \mathrm{w} / \mathrm{w}$ (heating rate $=20^{\circ} \mathrm{C}$ under nitrogen flow).

$50 \%$ by weight after MPEG- $b$-PDLL decomposition. This indicating that the weight ratio of MPEG- $b$-PDLL/ MPEG- $b$-PCL was about $50 / 50$ by weight corresponded to the feed blend ratio ( $1 / 1$ by weight). The TG results supported that the blend nanoparticles with different blend ratios can be prepared according to the ${ }^{1} \mathrm{H}-\mathrm{NMR}$ analysis, as described above.

Thermal stability results can be clearly determined from the derivative TG (DTG) thermograms, as shown in Figure 5. The peak of DTG thermogram indicate a temperature of maximum decomposition rate $\left(T_{d, \max }\right)$. The resulting $T_{d, \max }$ are also reported in Table $\mathbf{1}$. The $T_{d, \max }$ values of MPEG- $b$-PDLL and MPEG- $b$-PCL were 367 and $420^{\circ} \mathrm{C}$, respectively supported the higher thermal stability of MPEG- $b$-PCL than that of MPEG- $b$-PDLL. The DTG thermograms of blend nanoparticles exhibited two peaks (two $T_{d, \max }$ ) of MPEG- $b$-PDLL and MPEG$b$-PCL. It should be noted that the peak areas of DTG thermograms depended on the blend ratios. The peak area of the first peak of MPEG- $b$-PDLL decomposition steadily decreased as the MPEG- $b$-PDLL blend ratio decreased. The $T_{d, \max }$ of MPEG- $b$-PCL in the blend nanoparticles did not change. While, the $T_{d \text {, max }}$ of MPEG$b$-PDLL in the blend nanoparticles decreased slightly with the MPEG- $b$-PDLL blend ratio. The DTG results also supported that the blend nanoparticles with different blend ratios exhibited difference in thermal decomposition behaviors.

\section{Conclusions}

The MPEG- $b$-PDLL/MPEG- $b$-PCL blend nanoparticles with spherical in shape and smooth surface were successfully prepared by nano-precipitation method. Blend ratios of MPEG- $b$-PDLL/MPEG- $b$-PCL affects the ave-

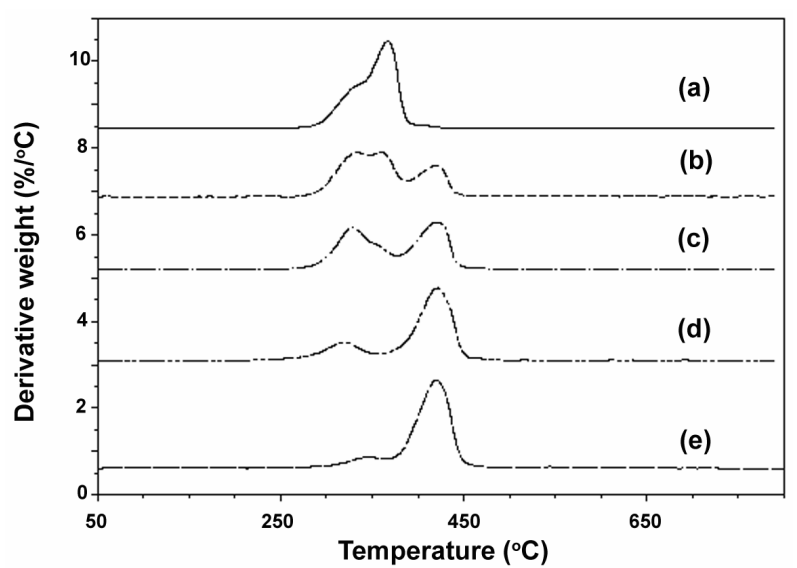

Figure 5. DTG thermograms of blend nanoparticles with MPEG- $b$-PDLL/MPEG- $b$-PCL blend ratios of (a) 4/0, (b) $3 / 1$, (c) $2 / 2$, (d) $1 / 3$ and (e) $0 / 4 \mathrm{w} / \mathrm{w}$ (heating rate $=10^{\circ} \mathrm{C}$ under nitrogen flow).

rage particle size, thermal transition properties and thermal decomposition behaviors. The average particle sizes and glass transition temperatures of the blend nanoparticles decreased and the heats of melting significantly increased as the blend ratio of MPEG- $b$-PCL increased. The blend ratio is an effective tool to modulate the particle size and thermal properties of the blend nanoparticles. The thermal property control suggested in this work may lead to rational manipulation of biodegradation and drug release rates of the blend nanoparticles. These blend nanoparticles might be interested in drug delivery applications.

\section{Acknowledgements}

The authors would like to acknowledge the financial support from the Mahasarakham University and the Research, Development and Engineering (RD\&E) fund through The National Nanotechnology Center (NANOTEC), The National Science and Technology Development Agency (NSTDA), Thailand (Project No. NN-B22-EN4-30-52-11) to Mahasarakham University. We also gratefully acknowledge The Center of Excellence for Innovation in Chemistry (PERCH-CIC), Commission on Higher Education, Ministry of Education, Thailand for providing a scholarship for one of us (W. Nanthakasri).

\section{REFERENCES}

[1] S. Y. Kim, Y. M. Lee and J. S. Kang, "IndomethacinLoaded Methoxy Poly(Ethylene Glycol)/Poly(D,L-Lactide) Amphiphilic Diblock Copolymeric Nanospheres: Pharmacokinetic and Toxicity Studies in Rodents,” Journal of Biomedical Material Research, Vol. 74A, No. 4, 2005, pp. 581-590. doi:10.1002/jbm.a.30342

[2] E. Pierri and K. Avgoustakis, "Poly(Lactide)-Poly(Ethyl- 
ene Glycol) Micelles as a Carrier for Griseofulvin,” Journal of Biomedical Material Research, Vol. 75A, No. 3, 2005, pp. 639-647. doi:10.1002/jbm.a.30490

[3] H. M. Aliabadi, A. Mahmud, A. D. Sharifabadi and A. Lavasanifar, "Micelles of Methoxy Poly(Ethylene Oxide)- $b$-Poly( $\varepsilon$-Caprolactone) as Vehicles for the Solubilization and Controlled Delivery of Cyclosporine A," Journal of Controlled Release, Vol. 104, No. 2, 2005, pp. 301-311. doi:10.1016/j.jconrel.2005.02.015

[4] X. W. Wei, C. Y. Gong, M. Gou, S. Z. Fu, Q. Guo, S. Shi, F. Luo, G. Guo, L. Y. Qiu and Z. Y. Qian, "Biodegradable Poly( $\varepsilon$-Caprolactone)-Poly(Ethylene Glycol) Copolymers as Drug Delivery System," International Journal of Pharmaceutics, Vol. 381, No. 2, 2009, pp. 1-18. doi:10.1016/j.ijpharm.2009.07.033

[5] R. Gref, Y. Minamitake, M. T. Peracchia, V. Trubetskoy, V. Torchilin and R. Langer, "Biodegradable Long-Circulating Polymeric Nanospheres,” Science, Vol. 263, No. 5153, 1994, pp. 1600-1603. doi:10.1126/science. 8128245

[6] S. Stolnik, L. Illum and S. S. Davis, "Long Circulating Microparticulate Drug Carriers," Advanced Drug Deliverly Review, Vol. 16, No. 2, 1995, pp. 195-214. doi:10.1016/0169-409X(95)00025-3

[7] S. Y. Kim, I. L. Shin, Y. M. Lee, C. S. Cho and Y. K. Sung, "Methoxy Poly(Ethylene Glycol) and $\varepsilon$-Caprolactone Amphiphilic Block Copolymeric Micelle Containing Indomethacin. II. Micelle Formation and Drug Release Behaviours,” Journal of Controlled Release, Vol. 51, No. 1, 1998, pp. 13-22.

[8] A. Lucke, J. Tebmar, E. Schnell, G. Schmeer and A. Gopferich, "Biodegradable Poly(D,L-Lactic Acid)-Poly (Ethylene Glycol)-Monomethyl Ether Copolymers: Structures and Surface Properties Relevant to Their Use as Biomaterials,” Biomaterials, Vol. 21, No. 23, 2000, pp. 2361-2370. doi:10.1016/S0142-9612(00)00103-4

[9] C. He, J. Sun, C. Deng, T. Zhao, M. Deng, X. Chen and $\mathrm{X}$. Jing, "Study of the Synthesis, Crystallization, and Morphology of Poly(Ethylene Glycol)-Poly( $\varepsilon$-Caprolac- tone) Diblock Copolymers,” Biomacromolecules, Vol. 5, No. 5, 2004, pp. 2042-2047. doi:10.1021/bm049720e

[10] H. Hyun, M. S. Kim, S. C. Jeong, Y. H. Kim, S. Y. Lee and H. B. Lee, "Preparation of Diblock Copolymers Consisting of Methoxy Poly(Ethylene Glycol) and Poly( $\varepsilon$ Caprolactone)/Poly(L-Lactide) and Their Degradation Property,” Polymer Engineering and Science, Vol. 46, 2006, pp. 1242-1249. doi:10.1002/pen.20581

[11] Y. Zhang, C. Wang, W. Yang, B. Shi and S. Fu, "TriComponent Diblock Copolymers of Poly(Ethylene Glycol)-Poly( $\varepsilon$-Caprolactone-Co-Lactide): Synthesis, Characterization and Loading Camptothecin," Colloid and Polymer Science, Vol. 283, No. 11, 2005, pp. 1246-1252. doi:10.1007/s00396-005-1306-5

[12] Y. H. Na, Y. He, X. Shuai, Y. Kikkawa, Y. Doi and Y. Inoue, "Compatibilization Effect of Poly( $\varepsilon$-Caprolactone)-b-Poly(Ethylene Glycol) Block Copolymers and Phase Morphology Analysis in Immiscible Poly(Lactide)/ Poly( $\varepsilon$-Caprolactone) Blends,” Biomacromolecules, Vol. 3, No. 6, 2002, pp. 1179-1186. doi:10.1021/bm020050r

[13] F. Ahmed and D. E. Discher, "Self-Porating Polymersomes of PEG-PLA and PEG-PCL: Hydrolysis-Triggered Controlled Release Vesicles," Journal of Controlled Release, Vol. 96, No. 1, 2004, pp. 37-53. doi:10.1016/j.jconrel.2003.12.021

[14] Y. Dong and S. S. Feng, "Nanoparticles of Poly(D,LLactide)/Methoxy Poly(Ethylene Glycol)-Poly(D,L-Lactide) Blends for Controlled Release of Paclitaxel," Journal of Biomedical Material Research, Vol. 78A, 2006, pp. 12-19. doi:10.1002/jbm.a.30684

[15] S. A. Casarin, S. M. Malmonge, M. Kobayashi and J. A. M. Agnelli, "Study on in Vitro Degradation of Bioabsorable Polymers Poly(Hydroxybutyrate-Co-Valerate)-PHBV and Poly(Caprolactone)-PCL," Journal of Biomaterials and Nanobiotechnology, Vol. 2, 2011, pp. 207-215. doi:10.4236/jbnb.2011.23026 